\title{
Allergy and immunity to fungal infections and colonization
}

\author{
R. Crameri, K. Blaser
}

\begin{abstract}
Allergy and immunity to fungal infections and colonization. R. Crameri, K. Blaser. C) ERS Journals Ltd 2002

ABSTRACT: Innate and cell-mediated immunity are considered as the principal defence lines against fungal infections in humans. Most opportunistic mycoses occur in individuals with defective innate and/or adaptive cellular immunity. The morbidity and mortality rates associated with infections caused by fungal pathogens are high, and prevention, diagnosis and treatment of these infections remain quite difficult.

A variety of pathological conditions, including impaired immune function, are believed to cause host susceptibility to fungal infections as well as to determine the severity and characteristics of the associated pathology. Nonspecific cellular immunity, mediated by macrophages, neutrophils and natural killer cells, provides efficient protection against fungal infections in healthy individuals. A major reason for the increase in systemic mycoses is undoubtedly related to an increased number of patients with congenital or acquired immunodeficiencies.

However, there is increasing clinical and experimental evidence indicating that antigen-specific cellular immunity may also play a critical role in host protection against fungi. A better understanding of reciprocal regulation between innate, humoral and adaptive immune responses in the development of an optimal antifungal immunity and, in particular, the improved definition of fungal antigens, may lead to a clarification of the mechanisms involved in host immunity to fungal infections.

Molecular cloning and characterization of fungal antigens reveals the involvement of related cross-reactive molecular structures produced by different fungi as pathological molecules involved in development of allergic reactions.

Eur Respir J 2002; 19: 151-157.
\end{abstract}

Swiss Institute of Allergy and Asthma Research (SIAF), Davos, Switzerland.

Correspondence: R. Crameri

Swiss Institute of Allergy and

Asthma Research (SIAF)

Obere Strasse 22

CH-7270 Davos

Switzerland

Fax: 41814100840

Keywords: Allergens

cellular and humoral immunity

fungal allergy

fungal infections

fungi

molecular cloning

Received: March 222001

Accepted after revision May 82001

This work was supported by the Swiss National Science Foundation, grants numbers 31-50515.97 and 31-63381.00.
Endemic and opportunistic fungal infections are considered as an important clinical problem, concomitantly with increasing numbers of patients undergoing immunosuppresive medical treatment [1] and with an increase in congenital and acquired immunodeficiencies [2]. The morbidity and mortality rates caused by normally harmless fungal species (e.g. Candida, Aspergillus, Fusarium and Trichosporum) are relatively high [3]. Moreover, diagnosis and treatment of these infections remain difficult and often unreliable. Fungal invasion in humans is associated with a wide variety of diseases, ranging from benign colonization, superficial skin infections and allergy, to life-threatening systemic mycoses [1].

Epidemiological data indicate that prolonged leucopenia and therapy with high doses of corticosteroids are major risk factors for the development of invasive mycoses $[4,5]$. Other types of immunodeficiencies, for example impaired T-cell function and humoral immunity, are not of themselves sufficient risk factors for the development of mycoses [6], but they do represent predominant risk factors for the development of opportunistic mycoses [1]. Accordingly, the investigation of a host's normal resistance to fungal infection, and a heightened awareness of fungal virulence factors in relation to specific immune defects, are clearly relevant in patients with mycoses. Such investigations are fundamental in understanding the reciprocal regulation between innate and adaptive cellular and humoral immunity, and consequently host resistance to fungal infections.

Exposure to fungal spores is ubiquitous and, therefore, of pivotal importance for mycoses acquired through the respiratory tract. Consequently, innate immunity plays a predominant role in clearance of inhaled spores [7]. Antibody production does not appears to contribute decisively to host defence [8]. Within the respiratory tract, the first line of defence against fungal spores is formed by the mucociliary clearance [7]. Remaining spores are ingested and killed

Previous articles in this Series: No. 1: Pilette C, Ouadrhiri Y, Godding V, Vaerman J-P, Sibille Y. Lung mucosal immunity: immunoglobulin-A revisited. Eur Respir J 2001; 18: 571-588. No. 2: Lambrecht BN, Prins J-B, Hoogsteden HC. Lung dendritic cells and host immunity to infection. Eur Respir J 2001; 18: 692-704. No. 3: Moore BB, Moore TA, Toews GB. Role of T-B-lymphocytes in pulmonary host defences. Eur Respir J 2001; 18: 846-856. No. 4: Message SD, Johnston SL. The immunology of virus infection in asthma. Eur Respir J 2001; 18: 10131025 . 
by monocytes/macrophages [9]. Therefore, the innate nonadaptive mucosal defence in the airway represents a highly efficient primary defence system in the elimination of these micro-organisms. At this stage, independent of the cellular defence system, defence effector proteins mediate the protection through antimicrobial activity [10]. These defensive proteins belong mainly to the family of cationic defensins. Together with other enzymes, they constitute a protective environment in the lung [11]. In addition to physical barriers and activation of adaptive immune mechanisms involving alveolar macrophages, healthy individuals can call upon a strong and independent second line of defence, formed by neutrophilic granulocytes. They mainly attack hyphae, which are too large for ingestion. These, in turn, are killed by oxidative and nonoxidative mechanisms including different defensins. Each of these two defence systems is able alone to protect the host against large spores over long time periods. Yeasts and moulds can cause invasive disease only if both protective lines are overstrained. Within the skin and lung of immunocompetent hosts, antigen presentation by appropriate antigen-presenting cells (APCs) results from phagocytosis of intact spores, fungal particles or soluble antigens released from the growing fungus. The activation of $\mathrm{T}$ - and B-lymphocytes activates cellular and humoral immune responses, which are able to support the innate immunity [12].

\section{Fungal infections and pathogenicity}

Systemic mycoses can be divided into two groups based on their ability to infect immunocompetent hosts. Infectious fungi, the first group, are classified as primary pathogens. These include Coccidioides immitis, Histoplasma capsulatum, Blastomyces, and Paracoccidioides [1]. The second group includes the opportunistic pathogens such as Candida, Aspergillus, Cryptococcus, Trichosporon and Fusarium. These can cause invasive, deep infections only if additional specific predisposing risk factors, such as immune deficiency or other underlying severe disease, are

Table 1. - Diagnostic criteria of allergic bronchopulmonary aspergillosis (ABPA) ${ }^{\#}$

\section{Criteria}

1. Asthma

2. Current or previous pulmonary infiltrate

3. Elevated total serum IgE $1000 \mathrm{ng} \cdot \mathrm{L}^{-1}$

4. Peripheral blood eosinophilia $1000 \cdot \mathrm{mm}^{-3}$

5. Immediate skin reactivity to Aspergillus

6. Serum precipitins to Aspergillus

7. Elevated specific serum IgE and serum $\operatorname{IgG}$ to Aspergillus fumigatus

8. Central bronchiectasis

Ig: immunoglobulin. ${ }^{*}$ : according to reference [17]; outcome strongly dependent on the quality of the extract used. Not all of these features may be present in all cases of ABPA because they vary with the activity and stage of the disease. The minimal criteria for diagnosis are the presence of criteria $1,3,5$, and 7 . concomitantly present. In the past, most fungi could be assigned to these two categories. However, recent findings clearly show that these two categories do not sufficiently discriminate among these species. For example, Saccharomyces cerevisiae, considered to be the least dangerous fungal species, was found to be responsible for invasive, life-threatening infection in several cases [13]. Fusarium, a well-known plant pathogen, was also reported to cause severe infections in humans, particularly in leukaemic patients [14]. Furthermore, Penicillium marneffei, a fungus endemic in Asia and Japan, can cause disseminated fungal infection, especially in human immunodeficiency virus (HIV) patients [15]. Therefore, it appears that fungi, previously regarded as nonpathogenic and harmless in humans, can cause life-threatening mycoses and be devastating in immunocompromised patients. Nevertheless, the precise nature of the immune defence cascade to pathogenic fungi and moulds still needs clarification. The association of fungal infections with certain forms of immune adequacy will contribute significantly to the understanding of natural defence mechanisms against mycoses.

In addition to systemic and opportunistic mycoses, various fungi are associated with a large number of allergic disorders in humans that occur with increased prevalence $[1,16]$. Among these, allergic bronchopulmonary aspergillosis (ABPA), a life threatening hypersensitivity disease associated with $A$. fumigatus colonization of the bronchial airway, was described as a lung disease with defined clinical, serological, radiological and pathological features difficult to diagnose especially in patients suffering from cystic fibrosis (CF) [17]. The first four of the eight criteria proposed for the diagnosis of ABPA (table 1) do not cause significant problems to the experienced clinician; however, they are not sufficient for reliable diagnosis of the syndrome. Moreover, independent of priority, not all of these criteria and symptoms must be fulfilled. Therefore, to improve diagnosis, serology should clearly contribute in order to either confirm or exclude ABPA as suspected from the clinical signs. It is evident that skin-test reactivity and serological findings largely depend on the quality of the fungal extract applied; indeed, there are significant batchto-batch variations in the content and quality of allergenic components [18]. Recent progress in molecular cloning of A. fumigatus allergens has not only generated new diagnostic tools, but it has also clarified the molecular background and nature of ABPA [17-19]. In particular, molecular definition and recombinant production of major A. fumigatus allergens has enabled easy serological discrimination of ABPA from $A$. fumigatus-related allergic asthma by immunoglobulin (Ig)-E-specific detection techniques [18, 19]. Increased levels of serum IgE against the two allergens Asp $f 4$ and Asp $f 6$ clearly distinguishes ABPA from A. fumigatus-allergic asthma [20, 21] (table 2).

\section{The compromised host}

A major reason for the increase in systemic mycoses is undoubtedly related to immunodeficiency. There 
Table 2. - Serological discrimination of allergic bronchopulmonary aspergillosis (ABPA) from Aspergillus fumigatus sensitization by using recombinant $A$. fumigatus allergens ( $\mathrm{A}$ Asp $f$ )

\begin{tabular}{|c|c|c|c|c|c|}
\hline \multirow[t]{2}{*}{ Patient group } & \multirow[t]{2}{*}{ Patients $\mathrm{n}$} & \multicolumn{4}{|c|}{ Allergens } \\
\hline & & $\mathrm{r} A s p f 1^{\#}$ & $\mathrm{r} A s p f 3^{\#}$ & $\mathrm{r} \operatorname{Asp} f 4^{\top}$ & $\mathrm{r} A s p f 6^{\#}$ \\
\hline \multicolumn{6}{|l|}{ ABPA with } \\
\hline Allergic asthma & 60 & 83 & 88 & 80 & 55 \\
\hline $\mathrm{CF}$ & 20 & 100 & 100 & 80 & 70 \\
\hline \multicolumn{6}{|c|}{ A. fumigatus-sensitized with } \\
\hline Allergic asthma & 40 & 45 & 53 & 0 & 0 \\
\hline $\mathrm{CF}$ & 20 & 60 & 90 & 0 & 0 \\
\hline \multicolumn{6}{|l|}{ Controls } \\
\hline Healthy & 20 & 0 & 0 & 0 & 0 \\
\hline $\mathrm{CF}$-controls ${ }^{+}$ & 10 & 0 & 0 & 0 & 0 \\
\hline
\end{tabular}

Data are presented as percentages unless otherwise stated. CF: cystic fibrosis. ${ }^{*}$ : these allergens are used for the diagnosis of $A$. fumigatus sensitization. The overall sensitization to Asp $f 1$ and/or Asp $f 3$ was shown to be $86 \%$ in $A$. fumigatus-sensitized individuals with or without APA [17, 20] and reached $97.5 \%$ in the group of CF patients [21]; ${ }^{\text {: }}$ these allergens are highly specific for ABPA. They allow serological discrimination between ABPA and A. fumigatus allergy in A. fumigatus-sensitized allergic asthmatics, with a specificity of $100 \%$ and a sensitivity of $90 \%[17,20]$. In CF patients, the sensitivity reached $100 \%$ [21]; ${ }^{+}$: CF-patients without sensitization to A. fumigatus [21].

are an increasing number of people with a suppressed or weakened immune system due to underlying disease, or to medical treatment (e.g. as in organ transplant recipients). Indeed, an increased prevalence in invasive mycoses was observed in patients after bone marrow or lung transplantation [5], and in patients with leukaemia or other haematological malignancies leading to neutropenia and granulocytopenia $[4,22]$. Although infectious diseases caused by primary pathogens might become epidemic in endemic areas [23], it is the increase in opportunistic infections which generates serious health problems. In principle, the fungi have not changed their habitat, but the surrounding factors that enable a saprophyte to turn into a virulent pathogen have become more common (e.g. in oropharyngeal candidiasis (OPC)). Before the emergence of acquired immune deficiency syndrome (AIDS), OPC outbreaks were relatively rare and limited to infants, elderly people, individuals treated with inhaled steroids and immunocompromised patients. Nowadays, this disease is a common infection in HIV patients. It affects $\sim 90 \%$ of HIV-infected individuals, with a $60 \%$ recurrence rate within 3 months [24]. Extensive OPC has therefore become one of the clinical marker disease of HIV/AIDS infection. In recent years, more knowledge has been accumulated regarding the specific risks to particular patient groups and the human defence against fungal infection. For example, it has been shown that alveolar macrophages from humans treated with high doses of cortisone lose their capacity to control and kill $A$. fumigatus conidia growth in vitro [25]. This suppressive effect is highly specific to corticosteroids, since Cyclosporin A, a T-cell-suppressing agent, does not affect the killing of $A$. fumigatus by macrophages [26]. Despite increased understanding of the host-fungus interactions, further detailed studies are required to fully understand the host defence mechanisms against fungi. A particular challenge for the immune system is related to yeasts and moulds able to grow under different morphological conditions, depending on the local environment, and thus capable of escaping the lines of defence of the innate immunity.

\section{Host defence mechanisms of the lung}

Several factors can influence the host's specific immunity to fungal spores. In particular, it involves mucociliary clearance, activation of alveolar macrophages and neutrophilic granulocytes, as well as T-cell-mediated immune functions and humoral immunity (fig. 1).

\section{Mucus and mucociliary clearance}

The airway mucus varies in amount, composition and physical properties at different levels of the airways. The mucus constitutes a physical, chemical and biological barrier of secretory products from the mucus membrane [7]. The airway fluid contains glycoproteins including mucins, proteoglycans, lipids, secretory (s) IgA, lysozyme, peroxidase and other substances (e.g. surfactant). Surface liquids provide an efficient biological barrier by interacting with microorganisms, thereby preventing them from adhering to and migrating through the airway epithelium. Furthermore, destruction of the epithelial cell barrier is rapidly followed by repair mechanisms, resulting in the influx of serum proteins and extracellular matrix proteins to the lumen site of the epithelium [27]. The mucus layer of the airways facilitates the elimination of inhaled particles, including fungal spores. Mucociliary clearance is mediated by coordinated ciliary movement that transports the epithelial fluid, together with trapped material, out of the airways into the pharynx. The anatomical and functional features of mucociliary transport, combined with surface-lining liquid absorption along the tracheobronchial tree, are regarded as the basic mechanisms ensuring that mucus does not accumulate within the central airways as it is transported toward them from the periphery [28]. 


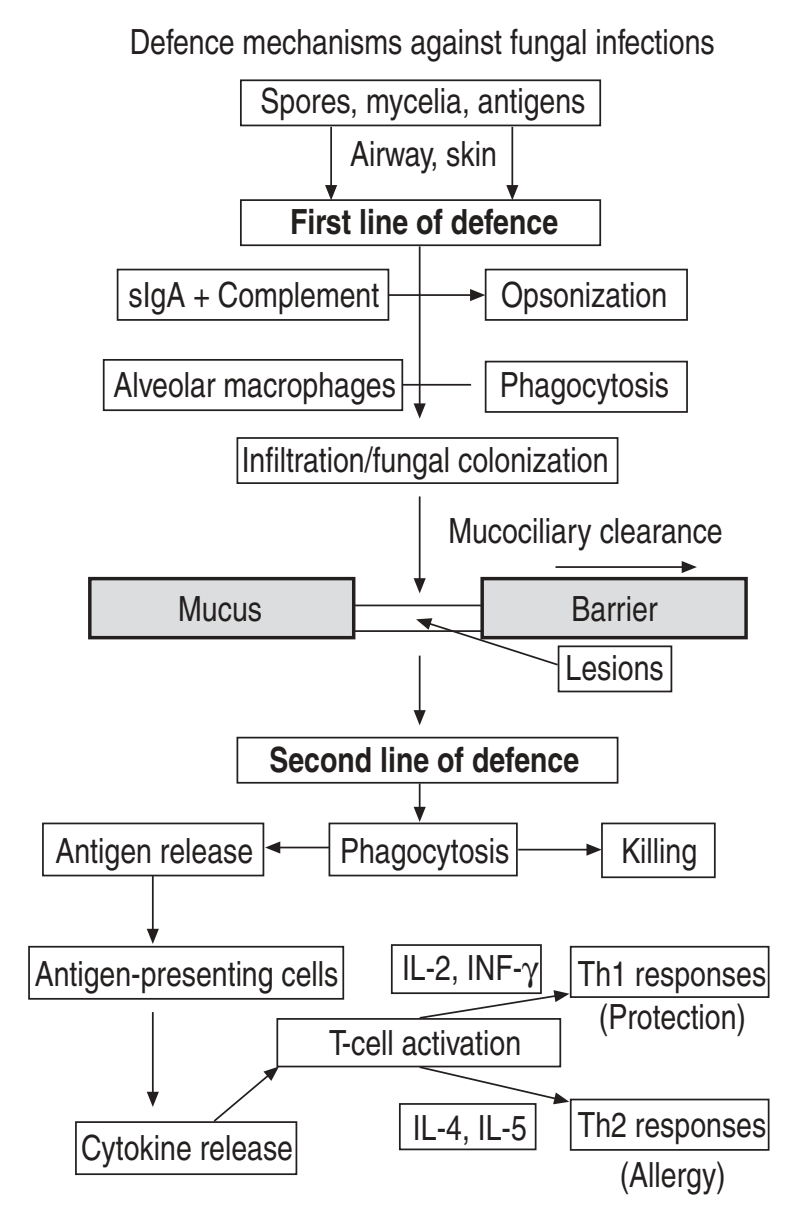

Fig. 1.-Flow of the immune response against fungi in healthy individuals. The first line of defence consisting of the mucus barrier and alveolar macrophages is generally sufficient for the efficient elimination of fungal spores. Breaching of the first line of defence may result in an increased exposure to fungal antigens eliciting a T-helper (Th)1-type response in normal individuals and in a Th2-type response in atopic individuals. $\operatorname{sgA}$ : secretory immunoglobulin-A; IL: interleukin; INF: interferon.

It is well established that physical and chemical injury impairs airway mucociliary function, and that various forms of airway disease are associated with mucociliary dysfunction. A good example is $\mathrm{CF}$, a genetic disease associated with a defective transmembrane regulator gene that leads to decreased chloride secretion into the airway lumen. Increased sodium and water re-adsorption from the lumen results in dehydrated, suboptimally-cleared mucus. CF patients are prone to bronchopulmonary colonization with bacteria and suffer from a high incidence of $A$. fumigatus colonization, correlated with disturbed neutrophilic functions [29].

\section{Alveolar macrophages and neutrophils}

Phagocytosis and killing by phagocytic cells constitute essential innate defence functions of the airways against micro-organisms, similar to the functions of polymorphonuclear cells and macrophages present in the mucus. Macrophages selectively protect against fungal spores that have escaped mucociliary clearance. Their importance in mediating the first line of host defence against inhaled microorganisms has been clearly demonstrated. In vivo studies in immunodeficient mice have shown that resident pulmonary macrophages are responsible for clearing the lung of inhaled conidia from A. fumigatus by a first-order kinetic mechanism [30]. The phagocytic cells bear surface receptors that can recognize surface structures of the micro-organisms in a nonadaptive way, the so-called "pattern recognition receptors" [31]. A critical surface structure of fungi is fungal mannan, which is recognized by the mannanbinding protein present on phagocytic cells, allowing recognition and efficient phagocytosis even in the absence of opsonizing complement or Igs. After binding and phagocytosis, alveolar macrophages secrete pro-inflammatory cytokines, such as interleukin (IL)-1, IL-6, interferon (IFN)- $\gamma$, and tumour necrosis factor (TNF)- $\alpha$, to prime and activate different cells in the environment. These cytokines are able to augment the phagocytic and killing capacity of phagocytes through enhanced oxygen freeradical production. Moreover, IL-1, TNF- $\alpha$, and INF- $\gamma$ secreted at the site of infection activate epithelial cells, resulting in additional production of chemokines and pro-inflammatory cytokines [32]. This induces a second wave of phagocyte recruitment in order to eliminate the micro-organisms from the infected site. Complement components deposited on the surface of micro-organisms at the site of inflammation may enhance their phagocytosis and killing. The importance of complement activation, particularly of the opsonic component C3, was documented in the antifungal defence [33]. In contrast, $\operatorname{SIgA}$ is produced as the result of a specific adaptive immune response. Binding of $\operatorname{sIgA}$ to the fungal surface prevents binding of spores to the epithelial surface, thus facilitating mucociliary clearance. Moreover, it was demonstrated both in vitro and in vivo that monocytes, alveolar macrophages and polymorphonuclear cells are able to phagocytose fungal spores. Conidia that have escaped the first line of cellular defence may germinate and grow in the hyphal form. There is experimental evidence that neutrophilic granulocytes provide reliable protection against hyphae. Indeed, neutropenia is considered the strongest predisposing factor for invasive candidiasis and invasive aspergillosis [5]. Moreover, it has been demonstrated that mononuclear cells and polymorphonuclear phagocytes provide selective protection against $A$. fumigatus conidiae and mycelia, respectively [9]. Adhesion by these cells to hyphae too large for phagocytosis, together with the release of toxic oxygen radicals and cationic peptides, appear to be key events that cause hyphal damage. In addition to prolonged neutropenia, chronic septic granulomatosis, a disease with a hereditary defect of oxygen free-radical formation by phagocytes, is associated with an enhanced risk for invasive fungoses, thus illustrating the importance of neutrophil-derived reactive oxygen intermediates in the control of fungal infections [5, 9]. 


\section{Cell-mediated adaptive immune functions}

Nonspecific cellular immunity, mediated by macrophages and neutrophils, is considered to provide the first two lines of defence against fungi and is part of the innate resistance [1]. Cell-mediated immunity provided by different T-helper (Th) cell subsets offers new perspectives for understanding the adaptive immunity to fungal infections [12]. The mechanisms of innate resistance and adaptive immunity are interdependent. Cellular interactions through cytokines, antibodies, complement and their corresponding surface receptors represent communication elements in the cross-talk between the two types of pathogenic resistance [34]. Fungal growth of spores in the lung or the skin produces fungal particles and soluble antigens that are presented by appropriate APCs as a consequence of phagocytosis. This activates specific T-cells and cytokine secretion, leading to antibody production by B-cells. Thus, cellular and humoral immune responses are generated. Depending on an individual's immunological status, either an equilibrated cytokine profile or a predominantly Th1- or Th2-like cytokine pattern may develop. Particular cytokines, such as IL-4 or IL-12, prime the T-cells either to differentiate into IL-4, IL-5 and IL-6, producing Th2 cells that favour allergic inflammatory responses and humoral immunity, or into IL-2 and IFN- $\gamma$, producing Th1 cells, favouring cell-mediated responses and the production of opsonizing antibodies.

\section{Protective and nonprotective $T$-cell responses to fungi}

Innate and cell-mediated immune responses form the most important protective mechanisms against opportunistic fungal pathogens. Clinical evidence to support this claim can be derived from subjects with primary or acquired cell-mediated immune deficiencies as well as those who are particularly susceptible to fungal infections [35]. Moreover, treatment with drugs that suppress cell-mediated immunity, such as corticosteroids or other immunosuppressive agents, is recognized as a major predisposing factor for fungal diseases [26]. Within this framework, experimental observation supports the protective role of Th1 cells to fungal infection, as opposed to Th2 responses. Acquired immunity to Candida albicans in oropharyngeal candidiasis is believed to prevent the mucosal colonization of immunocompetent individuals from progressing to symptomatic infection. Resistance to Candida-related disease correlates with cell-mediated immunity in vivo as well as Th1 cytokine secretion in vitro [36]. However, clinical observations suggest that in several disease states, including symptomatic infections, Candida antigens may trigger Th2-type cell reactivity.

\section{Humoral immunity}

The immunological significance of antibodies directed against circulating fungal antigens, as related to the development of protective immunity and/or pathology in fungal infections, is not yet completely understood. It has been shown that the great majority of patients suffering from chronic bronchitis, as well as many normal, healthy individuals, develop serum IgG against thermophilic actinomycetes and fungal antigens. However, although the presence of these antibodies is evidence of exposure, their role in the pathogenesis of respective diseases is not entirely clear [37]. Nonetheless, recent data strongly support the existence of protective antibodies against Candida and Cryptococcus, two of the major opportunistic fungal infections [38]. Moreover, inhibition of adhesion to the host's receptor cells, inhibition of germ-tube formation, opsonization and neutralization of virulence enzymes were proposed as key events in the protection. In humans, fungal infections can also be associated with various allergic disorders characterized by high IgE responses [16]. In atopic individuals, fungus-related obstructive airway diseases can be differentiated, on the one hand, by the development of asthmatic attacks due to transient high exposure to fungal spores resulting in Th2-type response, IgEmediated reaction and eosinophilic inflammation. On the other hand, a more complex asthmatic reactions resulting from colonization of the mucus-epithelial surface by virulent fungi, which is characterized by an exaggerated immunological response with intense eosinophil infiltration of the airways. Specific and total serum IgE levels directed against Aspergillus antigens are elevated in ABPA. In addition, peripheral and lung eosinophilia were detected together with increased specific CD4+ Th2 cell frequency [39]. Allergen-specific T-cells from patients with ABPA express a typical Th2-type cytokine pattern, with high IL-4 and little or no IFN- $\gamma$. However, IgE responses in aspergillosis result in a complicated and patientspecific sensitization pattern directed against various fungal allergens [17-20]. Cloning, characterization and production of recombinant $A$. fumigatus allergens enabled the discovery of specific IgE antibody production against disease-specific allergens and, in particular, to distinct allergens strictly specific for ABPA $[19,21]$. Serological investigations, involving $A$. fumigatus-sensitized patients with asthma and/or CF and suffering from ABPA, showed that the allergens Asp $f 1$ and Asp $f 3$ together bind serum $\operatorname{IgE}$ of all patients (table 2). However, the specific IgE levels to Asp $f 1$ and $A s p f 3$ did not discriminate between these diseases. In contrast, the recombinant allergens Asp $f 4$ and $A s p f 6$ allowed a clear-cut serological diagnosis of ABPA in the respective patients (table 2). In all cases, strong, positive skin tests to defined allergen challenges correlated with the presence of allergenspecific serum IgE, which demonstrates the biological relevance of these allergens in vivo [19]. In addition to diagnostic applications, pure and clearly-defined recombinant allergens provide an excellent tool to study the pathogenic mechanisms of fungal diseases.

\section{Future approaches}

Modern molecular biology has clearly elucidated a whole array of fungal molecules involved in allergic 
reactions. The combination of the functional enrichment of complementary deoxyribonucleic acid (cDNA) libraries displayed on phage-surface and roboticbased screening has allowed the rapid generation of information on the repertoire of IgE-binding molecules in complex allergen extracts [40, 41]. Sequence information, obtained by the screening of libraries constructed with the cDNA of A. fumigatus, Coprinus comatus, Cladosporium herbarum, Malassezia furfur and $C$. albicans, has defined whole arrays of structurally-conserved, immunologically-cross-reactive allergens expressed by these fungi [40]. Among them, thioredoxin, cyclophilins, manganese-dependent superoxide dismutase and the acidic ribosomal $\mathrm{P}_{2}$ protein show a high degree of sequence identity to the related human proteins [42-44]. IgE reactivity to the corresponding human proteins could be demonstrated in individuals sensitized to the respective fungal proteins and to those suffering from ABPA or severe atopic dermatitis. In chronic inflammation, this type of autoimmune reaction probably contributes to tissue damage at the site of inflammation. These reactions need to be studied in detail to clarify their role in the pathogenesis of severe and chronic atopic disorders. It remains to be elucidated whether autoreactivity is as a result of molecular mimicry between fungal antigens and released (typically intracellularly) human proteins or as a result of bystander sensitization by epitope spreading. In this context, the detection of autoimmune reactivity described in some candidal diseases is certainly of importance [45].

Infection by pathogens or parasites can induce differential immune responses in infected individuals. To date, it has been unclear whether differential immunoglobulin-E responses in clinically related diseases can be elicited by a single pathogen. However, the authors' work with recombinant Aspergillus fumigatus allergens has demonstrated that a fungus can elicit different immunoglobulin-E responses in patients suffering either from allergic bronchopulmonary aspergillosis or from Aspergillus fumigatusrelated asthma. Specific sensitization to proteins in allergic bronchopulmonary aspergillosis suggests clear differences in the pathway of fungal exposure and immunological recognition by patients expressing different fungus-related disease. Further work devoted to the elucidation of the allergen repertoire of fungi will substantially contribute to a better understanding of fungal pathogenesis at the molecular level.

Acknowledgements. The authors are grateful to P. Schmid-Grendelmeier for comments and careful reading of the manuscript.

\section{References}

1. Murphy JW, Friedman H, Bendinelli M. Fungal Infections and Immune Responses. New York, Plenum Press, 1993.

2. American Thoracic Society. Fungal infection in HIVinfected persons. Am J Respir Crit Care Med 1995; 152: 816-822.
3. Levitz SM. Overview of host defences in fungal infections. Clin Infect Dis 1992; 14: Suppl. 1, 37-42.

4. Fridkin SK, Jarvis WR. Epidemiology of nosocomial fungal infections. Clin Microbiol Rev 1996; 9: 499-511.

5. Saral R. Candida and Aspergillus infections in immunocompromised patients: an overview. Rev Infect Dis 1991; 13: 487-492.

6. Denning DW, Folansbee SE, Scolaro M, Norris S, Edelstein H, Stevens DA. Pulmonary aspergillosis in the acquired immunodeficiency syndrome. $N$ Engl $J$ Med 1991; 324: 654-662.

7. Wanner A, Salathe M, O'Riordan TG. Mucociliary clearance in the airways. Am J Respir Crit Care Med 1996; 154: 1868-1902.

8. Waldorf AR. Pulmonary defence mechanisms against opportunistic fungal pathogens. In: Kurstak E, ed. Immunology of Fungal Diseases. Immunology Series 47. New York, Marcel Dekker, 1989; pp. 243-270.

9. Schaffner A, Douglas H, Braude A. Selective protection against conidia by mononuclear and against mycelia by polymorphonuclear phagocytes in resistance to Aspergillus. J Clin Invest 1982; 69: 617-631.

10. Agerberth B, Gruenwald J, Castaños-Velez E, et al. Antibacterial components in bronchoalveolar lavage fluid from healthy individuals and sarcoidosis patients. Am J Respir Crit Care Med 1999; 160: 283290.

11. Schonwetter BS, Stolzenberg ED, Zasloff MA. Epithelial antibiotics induced at sites of inflammation. Science 1995; 267: 1645-1648.

12. Romani $\mathrm{L}$. The $\mathrm{T}$ cell response against fungal infections. Curr Opin Immunol 1997; 9: 484- 490.

13. Polak A, Hartman PG. Antifungal chemotherapy - are we winning? Prog Drug Res 1991; 37: 181-269.

14. Melcher GP, McGough DA, Fothergill AW, Norris C, Rinaldi MG. Disseminated hyalohyphomycosis caused by a novel human pathogen, Fusarium napiforme. J Clin Microbiol 1993; 13: 1461-1467.

15. Sirisantha V, Sirisantha T. Disseminated Penicillium marneffei infection in human immunodeficiency infected children. Pediatr Infect Dis J 1995; 14: 935940.

16. Horner SE, Helbling A, Salvaggio JE, Lehrer SB. Fungal allergens. Clin Microbiol Rev 1995; 8: 161-179.

17. Crameri R. Recombinant Aspergillus fumigatus allergens: From the nucleotide sequences to clinical applications. Int Arch Allergy Immunol 1998; 115: 99-114.

18. Crameri R, Lidholm J, Grönlund H, Stüber D, Blaser K, Menz G. Automated specific IgE assay with recombinant allergens: evaluation of recombinant Aspergillus fumigatus allergen I in the Pharmacia CAP system. Clin Exp Allergy 1996; 26: 1411-1419.

19. Hemmann S, Menz G, Ismail C, Blaser K, Crameri R. Skin test reactivity to 2 recombinant Aspergillus fumigatus allergens in $A$. fumigatus-sensitized asthmatic subjects allows diagnostic separation of allergic bronchopulmonary aspergillosis from fungal sensitization. J Allergy Clin Immunol 1999; 104: 601-607.

20. Crameri R, Hemmann S, Ismail C, Menz G, Blaser K. Disease-specific recombinant allergens for the diagnosis of allergic bronchopulmonary aspergillosis. Int Immunol 1998; 10: 1211-1216.

21. Hemmann S, Nikolaizik WH, Schöni MH, Blaser K, Crameri R. Differential IgE recognition of recombinant Aspergillus fumigatus allergens by cystic fibrosis patients with allergic bronchopulmonary aspergillosis 
or Aspergillus allergy. Eur J Immunol 1998; 28: 11551160 .

22. Gerson SL, Talbot GH, Hurwitz S, Strom BL, Lusk EJ, Cassileth PA. Prolonged granulocytopenia: the major risk factor for invasive aspergillosis in patients with acute leukemia. Ann Intern Med 1984; 100: 345 351.

23. Goldman M, Johnson PC, Sarosi GA. Fungal pneumonias. The endemic mycoses. Clin Chest Med 1999; 20: 507-519.

24. Hood S, Denning DW. Treatment of fungal infections in AIDS. J Antimicrob Chemother 1996; 37: Suppl. B, 71-85.

25. Schaffner A, Douglas H, Braude AI, Davis CE. Killing of Aspergillus spores depends on the anatomical source of the macrophage. Infect Immun 1983; 42: 1109-1115.

26. Berenguer J, Allende MC, Lee JW, et al. Pathogenesis of pulmonary aspergillosis. Granulocytopenia versus Cyclosporin and methylprednisolone-induced immunosuppression. Am J Respir Crit Care Med 1995; 152: 1079-1086.

27. Persson CG, Erjefalt JS, Erjefalt I, Korsgren MC, Nilsson MC, Sundler F. Epithelial shedding - restitution as a causative process in airway inflammation. Clin Exp Allergy 1996; 26: 746-755.

28. Asmundsson T, Kilburn KH. Mechanisms of respiratory tract clearance. In: Dulfano MJ, ed. Sputum. Springfield, IL, Charles C. Thomas, 1973; pp. 107 125.

29. Regnis JA, Robinson M, Bailey DL, et al. Mucociliary clearance in patients with cystic fibrosis and in normal subjects. Am J Respir Crit Care Med 1994; 150: 66-71.

30. Schaffner A, Bouglas H, Braude A. Selective protection against conidia by mononuclear and against mycelia by polymorphonuclear phagocytes in resistance to Aspergillus: Observations on these two lines of defence in vivo and in vitro with human and mouse phagocytes. J Clin Invest 1982; 69: 617-631.

31. Medzhitov R, Janeway CA. Innate immunity: impact on the adaptive immune response. Curr Opin Immunol 1997; 9: 4-9.

32. Humbert M, Ying S, Corrigan C, et al. Bronchial mucosal expression of the genes encoding chemokines RANTES and MCP-3 in symptomatic atopic and nonatopic asthmatics: Relationship to the eosinophilactive cytokines interleukin (IL)-5, granulocyte macrophage-colony-stimulating factor, and IL-3. Am J Respir Cell Mol Biol 1997; 16: 1-8.
33. Murphy JW. Immunity to fungi. Curr Opin Immunol 1989; 2: 360-367.

34. Trinchieri G, Kubin M, Bellone G, Cassatella MC. Cytokine cross-talk between phagocytic cells and lymphocytes: relevance for differentiation/activation of phagocytic cells and regulation of adaptive immunity. J Cell Biochem 1993; 53: 301-308.

35. Warnock DS, Richardson MD, eds. Fungal Infections in the Compromised Patient. New York, John Wiley \& Sons, 1990.

36. Ausiello CM, Urbani F, Gessani S, Sapgnoli GC, Gomez MJ, Cassone A. Cytokine gene expression in human peripheral blood mononuclear cells stimulated by mannoprotein constituents from Candida albicans. Infect Immun 1993; 61: 4105-4111.

37. Burrell R, Rylander R. A critical review of the role of precipitins in hypersensitivity pneumonitis. Eur J Respir Dis 1981; 62: 332-343.

38. Cassone A, Conti S, De Bernardis F, Polonelli L. Antibodies, killer toxins and antifungal immunoprotection: a lesson from nature?. Immunol Today 1997; 18: 164-169.

39. Knutsen AP, Mueller KR, Levine AD, Chouhan B, Hutcheson PS, Slavin RG. Asp $f 1$ CD4+ TH2-like $\mathrm{T}$-cell lines in allergic bronchopulmonary aspergillosis. J Allergy Clin Immunol 1994; 94: 215-221.

40. Crameri R, Walter G. Selective enrichment and highthroughput screening of phage surface-displayed cDNA libraries from complex allergenic systems. Comb Chem High Throughput Screen 1999; 2: 63-72.

41. Crameri R, Kodzius R, Konthur Z, Lehrach H, Blaser $\mathrm{K}$, Walter G. Tapping allergen-repertoires by advanced cloning technologies. Int Arch Allergy Immunol 2001; 124: 43-47.

42. Appenzeller U, Mayer C, Menz G, Blaser K, Crameri $\mathrm{R}$. IgE-mediated reaction to autoantigens in allergic diseases. Int Arch Allergy Immunol 1999; 118: 193-196.

43. Crameri R, Faith A, Hemmann S, et al. Humoral and cell-mediated autoimmunity in allergy to Aspergillus fumigatus. J Exp Med 1996; 184: 265-270.

44. Mayer C, Appenzeller U, Seelbach H, et al. Humoral and cell-mediated autoimmune reactions to human acidic ribosomal $\mathrm{P}_{2}$ protein in individuals sensitized to Aspergillus fumigatus $\mathrm{P}_{2}$ protein. J Exp Med 1999; 189: 1507-1512.

45. Ashman RB, Papadimitriou JM. Production and function of cytokines in natural and acquired immunity to Candida albicans infection. Microbiol Rev 1995; 59: 646-672. 